\title{
Cancer incidence and mortality in Shandong province, 2012
}

\author{
Zhentao Fu ${ }^{1}$, Zilong Lu ${ }^{1}$, Yingmei $\mathrm{Li}^{2}$, Jiyu Zhang ${ }^{1}$, Gaohui Zhang ${ }^{1}$, Xianxian Chen ${ }^{1}$, Jie Chu ${ }^{1}$, Jie Ren ${ }^{1}$, \\ Haiyan Liu', Xiaolei Guo ${ }^{1}$ \\ ${ }^{1}$ Shandong Center for Disease Control and Prevention, Jinan 250014, China; 'the Second People's Hospital of Jinan, Jinan 250001, China \\ Correspondence to: Xiaolei Guo. Shandong Center for Disease Control and Prevention, Jinan 250014, China. Email: guoxiaolei@126.com.
}

\begin{abstract}
Objective: Population-based cancer registration data in 2012 from all available cancer registries in Shandong province were collected by Shandong Center for Disease Control and Prevention (SDCDC). SDCDC estimated the numbers of new cancer cases and cancer deaths in Shandong province with compiled cancer incidence and mortality rates.
\end{abstract}

Methods: In 2015, there were 21 cancer registries submitted data of cancer incidence and deaths occurred in 2012. All the data were checked and evaluated based on the National Central Cancer Registry (NCCR) criteria of data quality. Qualified data from 15 registries were used for cancer statistics analysis as provincial estimation. The pooled data were stratified by area (urban/rural), gender, age group $(0,1-4,5-9,10-14, \ldots, 85+$ years) and cancer type. New cancer cases and deaths were estimated using age-specific rates and corresponding provincial population in 2012. The Chinese census data in 2000 and Segi's population were applied for age-standardized rates. All the rates were expressed per 100,000 person-year.

Results: Qualified 15 cancer registries (4 urban and 11 rural registries) covered 17,189,988 populations (7,486,039 in urban and 9,703,949 in rural areas). The percentage of cases morphologically verified (MV\%) and death certificate-only cases (DCO\%) were $66.12 \%$ and $2.93 \%$, respectively, and the mortality to incidence rate ratio (M/I) was 0.60. A total of 253,060 new cancer cases and 157,750 cancer deaths were estimated in Shandong province in 2012. The incidence rate was 263.86/100,000 (303.29/100,000 in males, 223.23/100,000 in females), the age-standardized incidence rates by Chinese standard population (ASIRC) and by world standard population (ASIRW) were 192.42/100,000 and $189.50 / 100,000$ with the cumulative incidence rate (0-74 years old) of $22.07 \%$. The cancer incidence, ASIRC and ASIRW in urban areas were 267.64/100,000, 195.27/100,000 and 192.02/100,000 compared to 262.32/100,000, $191.26 / 100,000$ and $188.48 / 100,000$ in rural areas, respectively. The cancer mortality was $164.47 / 100,000$ (207.42/100,000 in males, 120.23/100,000 in females), the age-standardized incidence rates by Chinese standard population (ASMRC) and by world standard population (ASMRW) were 117.54/100,000 and 116.90/100,000, and the cumulative mortality rate (0-74 years old) was $13.53 \%$. The cancer mortality, ASMRC and ASMRW were $141.59 / 100,000,101.17 / 100,000$ and 100.33/100,000 in urban areas, and 173.79/100,000, 124.20/100,000 and 123.64/100,000 in rural areas, respectively. Cancers of the lung, stomach, liver, esophagus, colorectum, female breast, brain, leukemia, bladder and pancreas were the most common cancers, accounting for about $82.12 \%$ of all cancer new cases. Lung cancer, stomach cancer, liver cancer, esophageal cancer, colorectal cancer, female breast cancer, pancreatic cancer, brain tumor, leukemia and lymphoma were the leading causes of cancer death, accounting for about $89.01 \%$ of all cancer deaths. The cancer spectrum showed difference between urban and rural, males and females both in incidence and mortality rates.

Conclusions: Cancer surveillance information in Shandong province is making great progress with the increasing number of cancer registries, population coverage and the improving data quality. Cancer registration plays a fundamental role in cancer control by providing basic information on population-based cancer incidence, mortality, survival and time trend. The disease burden of cancer is serious in Shandong province, and so cancer prevention and control in Shandong province should be enhanced including health education, health promotion, cancer screening and cancer care services.

Keywords: Cancer registry; incidence; mortality; epidemiology; Shandong province 
Submitted Mar 08, 2016. Accepted for publication Mar 30, 2016.

doi: 10.21147/j.issn.1000-9604.2016.03.01

View this article at: http://dx.doi.org/10.21147/j.issn.1000-9604.2016.03.01

\section{Introduction}

Cancer is a leading cause of death worldwide in countries of all income levels $(1,2)$. In China, it is now the leading cause of death, causing about one fourth of all deaths (3). The Shandong Center for Disease Control and Prevention (SDCDC) is responsible for collecting population-based cancer registration data from local cancer registries for cancer statistics and publishing cancer registry annual report. The cancer registration data are not only widely used for cancer control and decision making, but also for scientific research (4). In this article, we provide a comprehensive overview of cancer incidence and mortality rates and estimate new cancer cases and cancer deaths in Shandong province in 2012.

\section{Materials and methods}

\section{Data source}

The SDCDC is in charge of population-based cancer registry with responsibility of data collection, evaluation and publication from local cancer registries in Shandong province. The cancer registration data reported to cancer registries were from local hospitals, community health service centers, the Basic Medical Insurances for Urban Residents and the New-Rural Cooperative Medical System. By June 1, 2015, 21 cancer registries (7 cities and 14 counties) submitted 2012 data to the National Central Cancer Registry (NCCR). The data covered about $22,628,604$ people, accounting for about $23.59 \%$ of the whole population in Shandong province at the end of 2012. All cancer cases were classified according to the International Classification of Diseases for Oncology, 3rd edition (ICD-O-3) and the International Statistical Classification of Diseases and Related Health Problems 10th Revision (ICD-10). There were 15 qualified registries finally accepted for the estimation of incidence and mortality in 2012.

\section{Population estimates}

The provincial population in 2012 was obtained from Public Security Bureau of Shandong Province and was estimated based on the fifth National Census data (2000) provided by the Statistics Bureau of Shandong province, taking into account of the changes of age composition, gender ratio and the proportion of urban and rural transformation released by the Statistics Bureau of Shandong Province (http://www.statssd.gov.cn/). The provincial population in 2012 was stratified by area (urban/rural), gender (male/female) and age groups $(0-, 1-4,5-84$ by 5 years, $85+$ years). The age-specific death probability was adjusted referring to the Six National Census in 2010. Linear changes were assumed in each age group between the fifth and sixth Population Census.

\section{Quality control}

The SDCDC checked and evaluated the quality and comparability of submitted data based on "Guideline for Chinese Cancer Registration" and referring to relevant data quality criterion of "Cancer Incidence in Five Continents Volume IX" by International Agency for Research on Cancer/ International Association of Cancer Registries (IARC/IACR) (5). The data included in the final analysis should meet the following criteria: the percentage of cases morphologically verified (MV\%) was not lower than $66 \%$; the percentage of death certificate-only cases (DCO \%) was lower than $15 \%$; the mortality to incidence ratio $(\mathrm{M} / \mathrm{I})$ was between 0.6 and 0.8 ; and the percentage of the diagnosis of unknown basis (UB\%) was lower than $5 \%$. IARC/IACR check software was used to determine whether all the variables are complete and valid $(6,7)$.

\section{Statistical analysis}

Incidence and mortality rates were calculated by area, gender and age groups. The numbers of new cases and deaths were estimated using the 5-year age-specific cancer incidence/ mortality rates and the corresponding populations. The Chinese population in 2000 and World Segi's population were used for age-standardized rates. The cumulative risk of developing or dying from cancer before 75 years of age (in the absence of competing causes of death) was calculated and presented as a percentage. Software including MS-Excel, IARCcrgTools 2.05 issued by IARC and IACR were used for data checking and evaluation (7). SAS software (SAS Institute 
Inc., Cary, USA) was used to calculate the incidence and mortality rates.

\section{Results}

\section{Data quality}

There were 15 registries involved in this analysis, including
4 cities and 11 counties covered 17,189,988 of populations (8,677,206 males and 8,512,782 females) and accounted for $17.92 \%$ of the whole population in Shandong province at the end of 2012. The overall indicators of $\mathrm{MV} \%, \mathrm{DCO} \%$, and $\mathrm{M} / \mathrm{I}$ ratio were $66.12 \%, 2.93 \%$ and 0.60 , respectively. They were $61.98 \%, 3.77 \%$ and 0.54 in urban registries, compared to $70.26 \%, 2.10 \%$ and 0.67 in rural registries. The quality evaluation for major cancers is presented in Table 1.

Table 1 Quality evaluation of cancer registration data in Shandong province, 2012

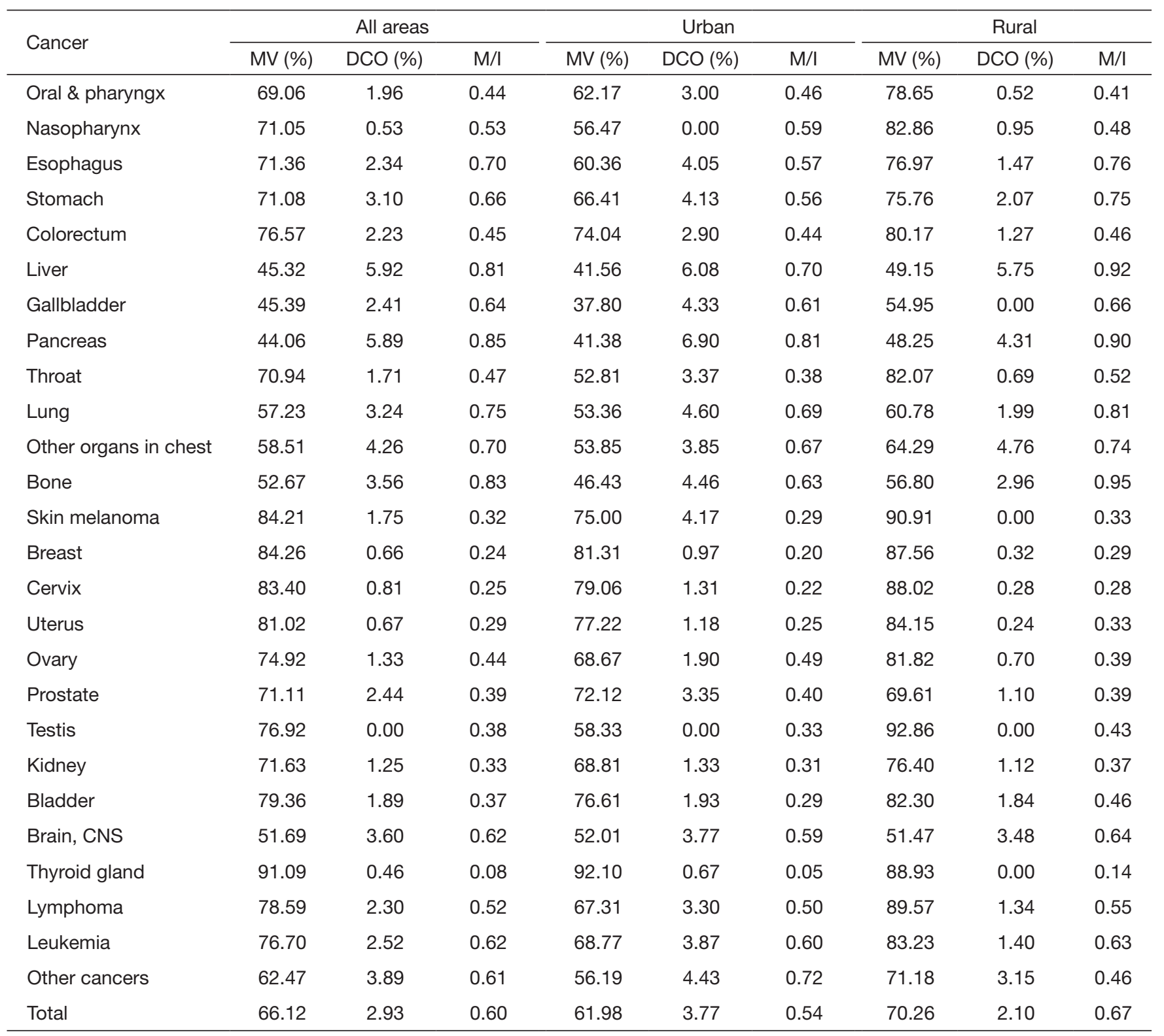

MV\%, percentage of morphological verification; DCO\%, percentage of cancer cases identified with death certification only; M/I, mortality to incidence ratio; CNS, central nervous system. 


\section{Incidence and mortality of overall cancers}

\section{Incidence}

It was estimated that there were 253,060 new cases diagnosed as cancer in 2012. The crude incidence rate of all cancers in Shandong province was 263.86/100,000 (303.29/100 000 in males, 223.23/100 000 in females). The age-standardized incidence rates by Chinese standard population (ASIRC) and by world standard population (ASIRW) were 192.42/100,000 and 189.50/100,000, respectively. Among the cancer patients aged $0-74$ years, the cumulative incidence rate was $22.07 \%$. The crude incidence rate and age-standardized rate in urban areas were similar to those in rural areas. The crude incidence rate and age-standardized rate in males and females were higher in urban areas than in rural areas (Table 2).

Table 2 Cancer incidence in Shandong province, 2012

\begin{tabular}{llccccc}
\hline Areas & Sex & Cases (thousand) & $\begin{array}{c}\text { Crude incidence } \\
\left(1 / 10^{5}\right)\end{array}$ & ASIRC $\left(1 / 10^{5}\right)^{\star}$ & ASIRW $\left(1 / 10^{5}\right)^{\star *}$ & $\begin{array}{c}\text { Cumulative rate } \\
0-74(\%)\end{array}$ \\
\hline \multirow{3}{*}{ All areas } & Both & 253.06 & 263.86 & 192.42 & 189.50 & 22.07 \\
& Male & 147.60 & 303.29 & 229.93 & 229.37 & 27.09 \\
& Female & 105.46 & 223.23 & 159.20 & 153.76 & 17.15 \\
\multirow{4}{*}{ Urban } & Both & 74.26 & 267.64 & 195.27 & 192.02 & 22.06 \\
& Male & 42.68 & 305.35 & 232.11 & 231.59 & 26.82 \\
& Female & 31.57 & 229.34 & 163.48 & 157.47 & 17.47 \\
\multirow{3}{*}{ Rural } & Both & 178.81 & 262.32 & 191.26 & 188.48 & 22.07 \\
& Male & 104.92 & 302.46 & 229.05 & 228.48 & 27.20 \\
& Female & 73.89 & 220.72 & 157.45 & 152.24 & 17.02 \\
\hline
\end{tabular}

*, age-standardized incidence rate (China population, 2000); **, age-standardized incidence rate (Segi's population).

\section{Age-specific incidence rate}

The age-specific incidence rate was relatively lower before 40 years old, then increased dramatically since then and peaked at age group of 80-84 years. The pattern was similar between urban and rural areas. With the increase of age, the changing trend of agespecific incidence rate was similar between urban and rural areas. Between age of 20-44 years, females had higher incidence rates than males both in urban and rural areas. While in age of over 45 years, the age-specific incidence rate of males was higher than that of females both in urban and rural areas (Figure 1, Table 3).

\section{Mortality}

It was estimated there were 157,750 died from cancer

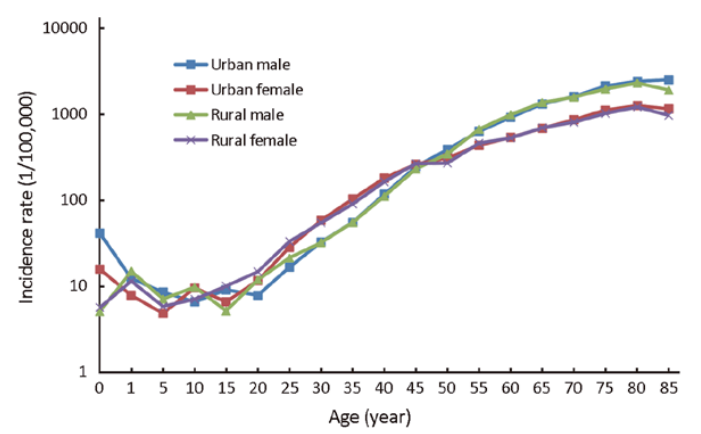

Figure 1 Age-specific cancer incidence rates in urban and rural areas, 2012. in 2012. The crude mortality of all cancers in Shandong province was $164.47 / 100,000$ (207.42/100,000 in males, $120.23 / 100,000$ in females). The age-standardized mortality rates by Chinese standard population (ASMRC) and by world standard population (ASMRW) were 117.54/100,000 and 116.90/100,000, respectively. Among the patients aged 0-74 years, the cumulative mortality rate was $13.53 \%$. The crude cancer mortality rate and age-standardized rate in rural areas were higher than those of urban areas. The crude cancer mortalities and age-standardized rate were higher in rural areas than in urban areas both for males and females (Table 4).

\section{Age-specific mortality rate}

The age-specific mortality rate was relatively lower before 45 years and then dramatically increased, reaching peak after 85 years. The mortality in rural areas was higher in age group of 20-84 years, while urban areas had higher mortalities after the age of 85 years. The mortality for females in rural areas was the highest in the age group of $80-84$ years. The age-specific mortality rate in urban males was lower than that in rural in most age groups (10-84 years). In females over 20 years old, the mortality in urban areas was higher than that in rural areas only for age group over 85 years (Figure 2, Table 5). 
Table 3 Age-specific incidence rates of overall cancers, $2012\left(1 / 10^{5}\right)$

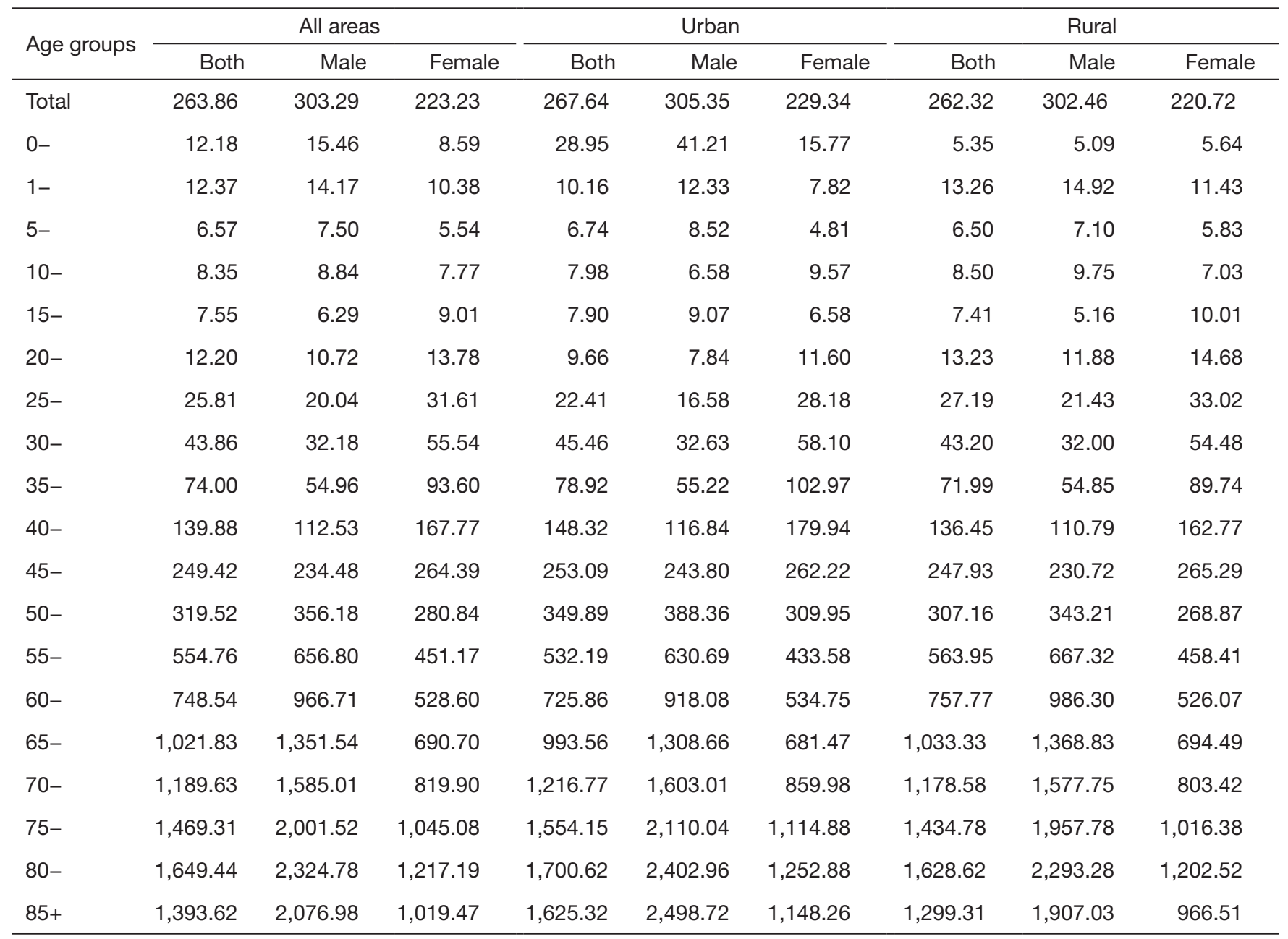

Table 4 Cancer mortality in Shandong province, 2012

\begin{tabular}{|c|c|c|c|c|c|c|}
\hline Area & Sex & Deaths (thousand) & Mortality $\left(1 / 10^{5}\right)$ & $\operatorname{ASMRC}\left(1 / 10^{5}\right)^{*}$ & $\operatorname{ASMRW}\left(1 / 10^{5}\right)^{\star \star}$ & Accumulated rate 0-74 (\%) \\
\hline \multirow{3}{*}{ All areas } & Both & 157.75 & 164.47 & 117.54 & 116.90 & 13.53 \\
\hline & Male & 100.95 & 207.42 & 157.54 & 157.44 & 18.19 \\
\hline & Female & 56.80 & 120.23 & 81.26 & 80.07 & 8.94 \\
\hline \multirow{3}{*}{ Urban } & Both & 39.29 & 141.59 & 101.17 & 100.33 & 11.16 \\
\hline & Male & 25.02 & 179.03 & 136.47 & 136.08 & 15.13 \\
\hline & Female & 14.26 & 103.59 & 69.71 & 68.47 & 7.30 \\
\hline \multirow{3}{*}{ Rural } & Both & 118.46 & 173.79 & 124.20 & 123.64 & 14.50 \\
\hline & Male & 75.92 & 218.87 & 166.03 & 166.05 & 19.43 \\
\hline & Female & 42.54 & 127.07 & 86.00 & 84.84 & 9.62 \\
\hline
\end{tabular}

*, age-standardized mortality rate (China population, 2000); **, age-standardized mortality rate (Segi’s population). 
Table 5 Age-specific mortality of overall cancers, $2012\left(1 / 10^{5}\right)$

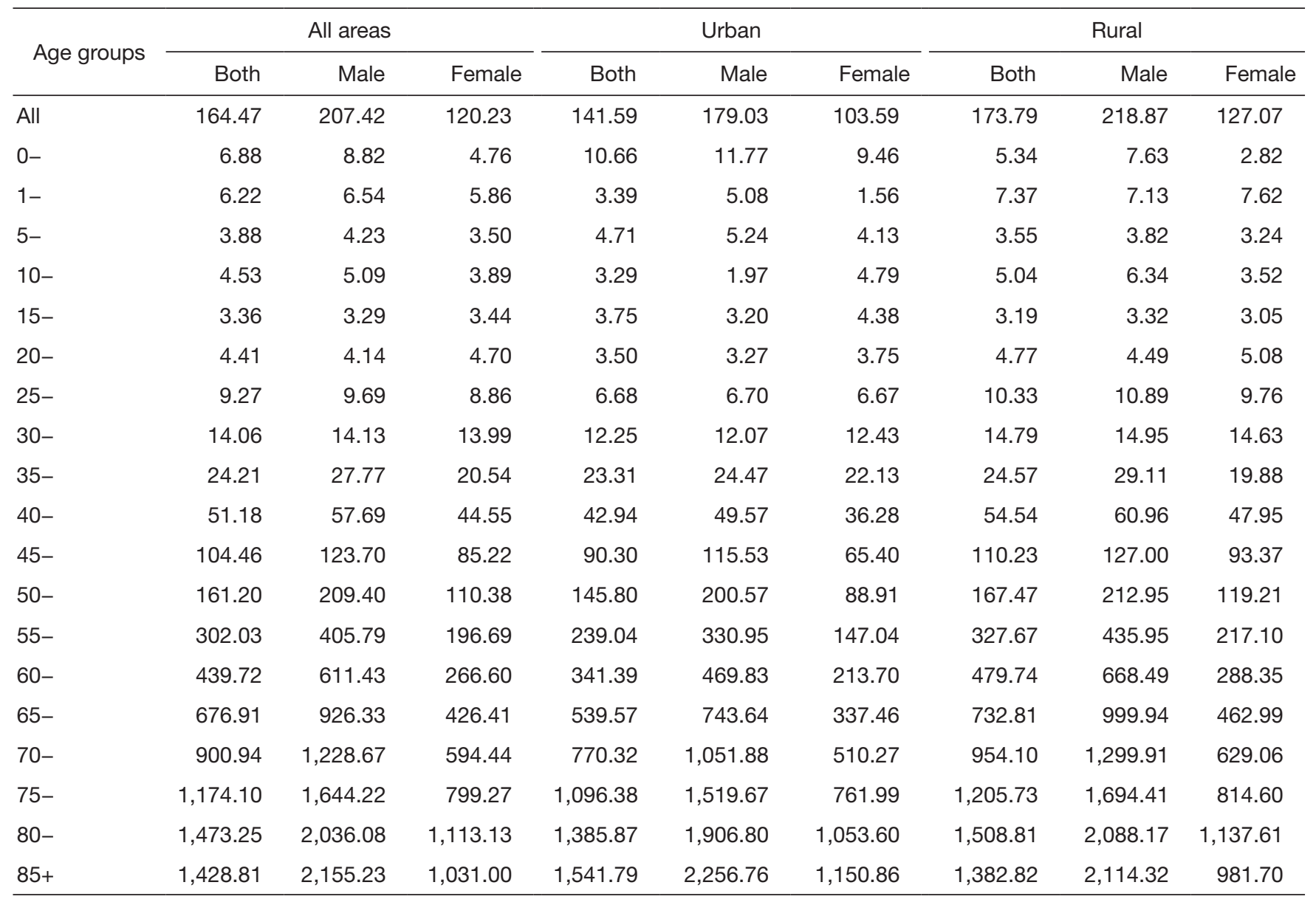

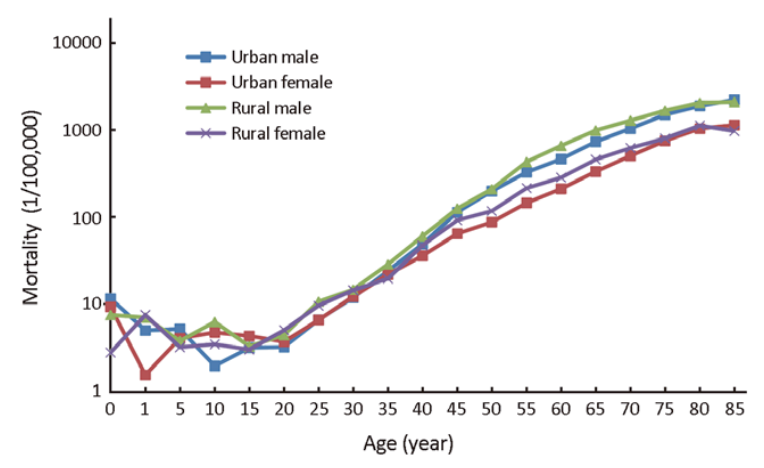

Figure 2 Age-specific cancer mortality in urban and rural areas, 2012 .

\section{Incidence and mortality for major cancers}

Cancer incidence for the $\mathbf{1 0}$ most common cancers

Lung cancer was the most common cancer in Shandong province, followed by stomach cancer, liver cancer, esophageal cancer and colorectal cancer, with estimated new cases of $59,200,36,800,27,140,24,340$ and 20,100, respectively. Lung cancer was the most frequently diagnosed cancers in males followed by stomach cancer, liver cancer, esophageal cancer and colorectal cancer. Lung cancer was also the most common cancers in females followed by breast cancer, stomach cancer, colorectal cancer and liver cancer (Table 6).

\section{Cancer death of the top 10 cancers}

Lung cancer was the leading cause of death in Shandong province, followed by stomach cancer, liver cancer, esophageal cancer and colorectal cancer with estimated deaths of 46,980, $25,340,23,220,17,520$ and 8,940, respectively. In males, lung cancer was the leading cause followed by stomach cancer, liver cancer, esophageal cancer and colorectal cancer; while in females, lung cancer was still the leading cause followed by stomach cancer, liver cancer, esophageal cancer and breast cancer (Table 7). 

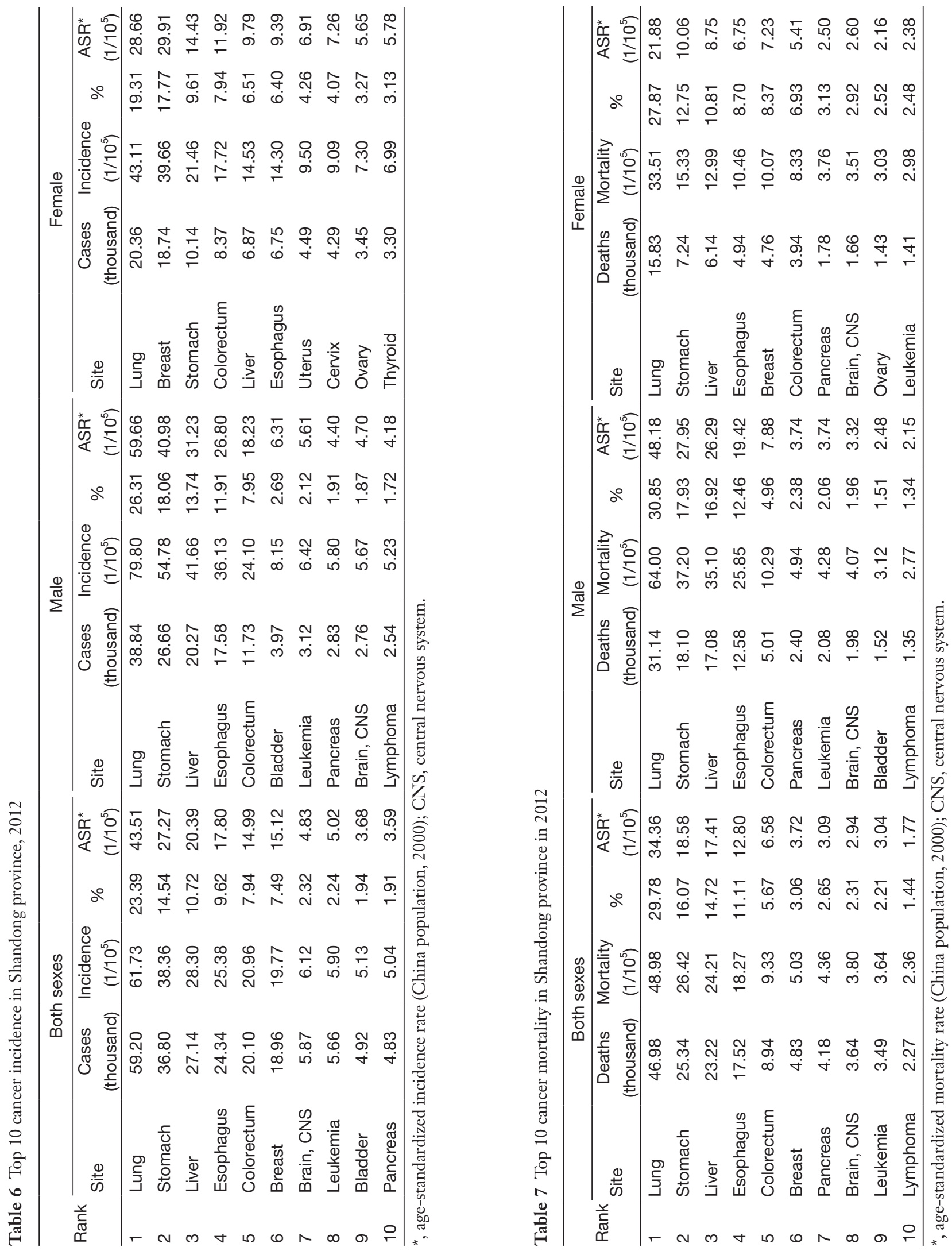
Cancer incidence of the 10 most common cancers in urban areas

In urban areas, lung cancer was the most frequently diagnosed cancer, followed by stomach cancer, liver cancer, colorectal cancer and female breast cancer with the estimated new cases of $16,300,10,810,8,030,7,460$ and 5,980, respectively. The most common sites of cancer were lung, stomach, liver, colorectum and esophagus in males, while in females, cancers of the breast, lung, colorectum, stomach and liver were the most common cancers (Table 8).

\section{Cancer death of the top 10 cancers in urban areas}

Lung cancer was the leading cause of cancer death in urban areas for both male and female with estimated number of deaths of 7,970 and 4,510, respectively. Other cancer types with high mortality in males were stomach cancer, liver cancer, colorectal cancer and esophageal cancer. In females, stomach cancer was also the second cause of cancer death, followed by liver cancer, colorectal cancer, and breast cancer (Table 9).

\section{Cancer incidence of the 10 most common cancers in rural areas}

Table 10 shows the 10 most common cancer incidence rates in rural areas. Lung cancer was the most frequently diagnosed cancer with estimated new cases of 42,900 and incidence rate of 62.94/100,000, followed by stomach cancer, esophageal cancer, liver cancer, and breast cancer. The most common sites of cancer in males were lung, stomach, liver, esophagus and colorectum, while in females they were lung, breast, stomach, esophagus and colorectum.

\section{Cancer death of the top $\mathbf{1 0}$ cancers in rural areas}

Lung cancer was the leading cause of cancer death in rural areas for both males and females. The number of deaths on lung cancer in rural areas was 34,500 with mortality of $50.61 / 100,000$. The other cancer types with high mortality were stomach cancer, liver cancer, esophageal cancer and colorectal cancer in males; while stomach cancer, liver cancer, esophageal cancer and breast cancer in females (Table 11).

\section{Discussion}

Measuring the burden of cancer in a population is essential for public health and cancer control. Reliable estimates of the cancer burden can provide a comprehensive picture of how the impact of cancer varies between geographic areas and between population strata (8). Shandong is the second most populous province in China, which has a population of more than 95 million, nearly two times of Korea. Since 2005, the SDCDC had established 7 cancer registries in the whole province. In the past few years, the number of cancer registry increased rapidly. In 2012, there were more than 20 cancer registries funded by the central finance of China.

In 2015, 21 cancer registries submitted registration data to the SDCDC. The population coverage of all registries was about 22.63 million, accounting for $23.59 \%$ of overall populations in Shandong province. In order to ensure the reliability of cancer registration data, stringent quality control process is used based on the national criteria issued in program protocol. The incidence, mortality and population from a same registry have to keep reasonable levels compared to past data. If the data are uncompleted, inexplicable or illogical, they would be sent back to the registries for check and modification. The indicators of completeness, comparability and invalidity, such as, $\mathrm{MV} \%, \mathrm{DCO} \%, \mathrm{M} / \mathrm{I}$ ratio, $\mathrm{UB} \%$ and percentage of cancer with undefined or unknown primary site (secondary) (O\&U\%), were evaluated for each registry's data to judge if submitted data are valid or not. There were 15 registries' data qualified for the update cancer incidence and mortality in 2012. These registries covered $17.92 \%$ of the provincial population at the end of 2012.

The estimated new cancer cases and deaths were 253,060 and 157,750 in 2012, respectively. The incidence and mortality were $263.86 / 100,000$ and $164.47 / 100,000$. Compared to the national data in 2010 (9) and 2011 (10), the crude cancer incidence and mortality rates in Shandong province were higher than those in China, after adjusted by age, the incidence and mortality rates were still higher. Compared with Korea (11), which is a neighbor of Shandong province with a linear distance less than $100 \mathrm{~km}$, although the population of Shandong was nearly 2 times that of Korea, but new cancer cases were lower than that in Korea $(253,060 / 280,556)$. The crude and age-standardized cancer incidences in Korea were 2.09 times (551.6/100,000 vs. 263.86/100,000) and 1.83 times $(347.6 / 100,000$ vs. 189.50/100,000) that of Shandong, respectively. While the crude cancer mortality and agestandardized cancer mortality in Korea were all lower than that in Shandong province of China. This indicated that measures taken to improve cancer survival rates and reduce mortality were also important.

Shandong province, similar to China (12), had higher incidence rate and lower mortality rate in urban areas than that in rural areas. But the cancer patterns in Shandong province had some differences with the whole country. Stomach cancer, instead of liver cancer, was the second cause of cancer deaths in Shandong province. Lung cancer was the most common 

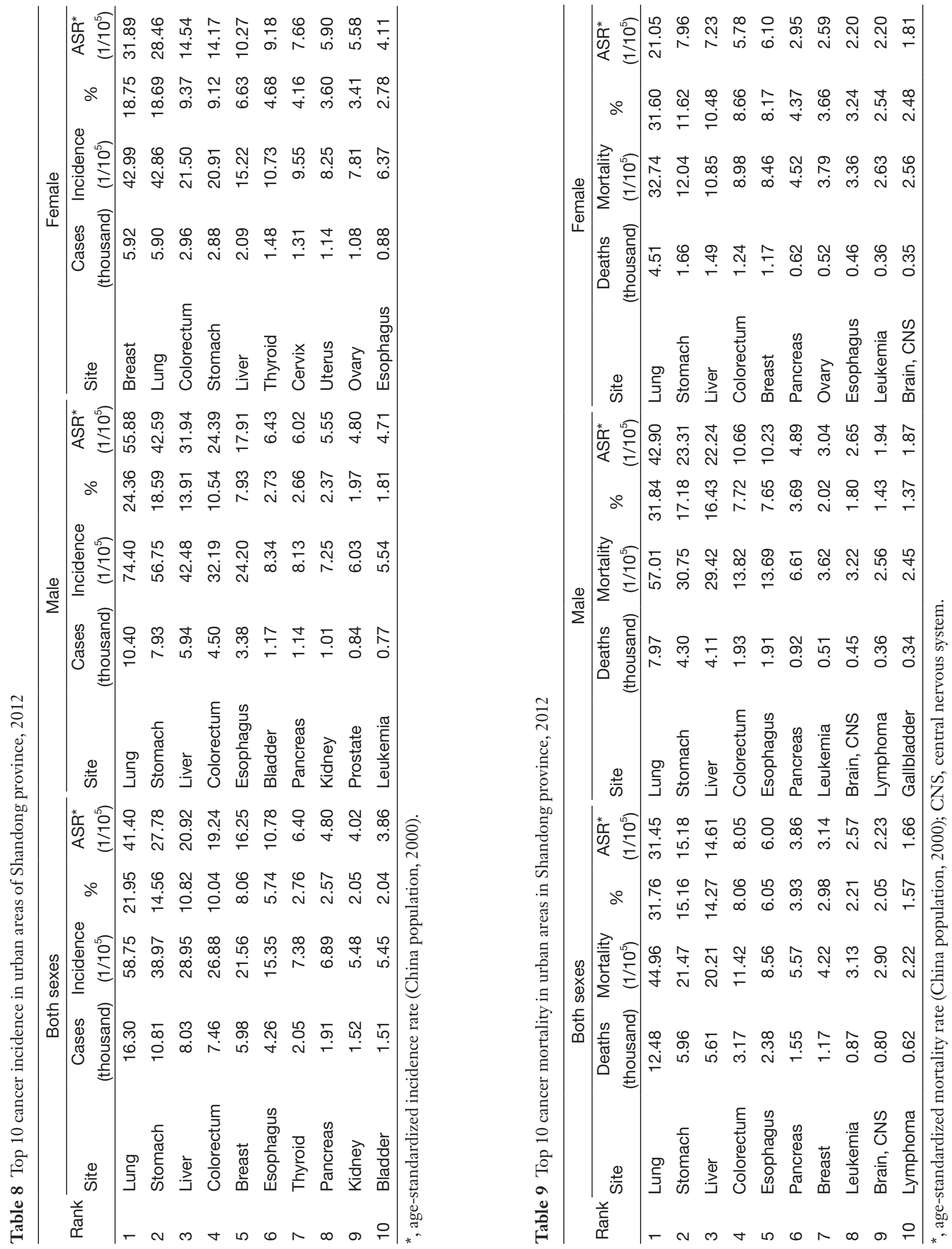
赵 幽

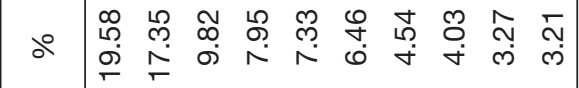

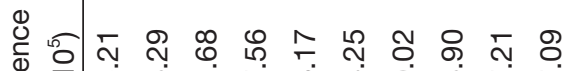

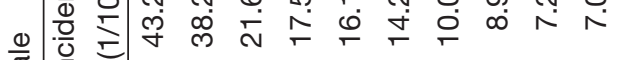

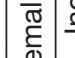
迎

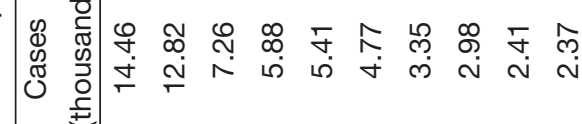

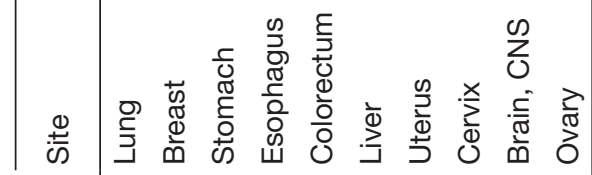
* एट

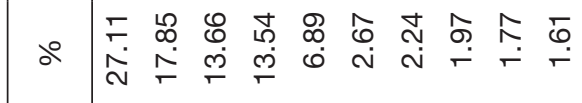

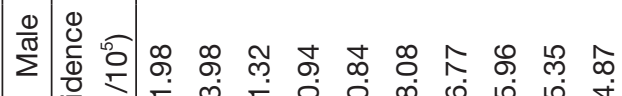

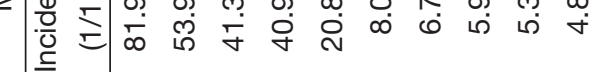

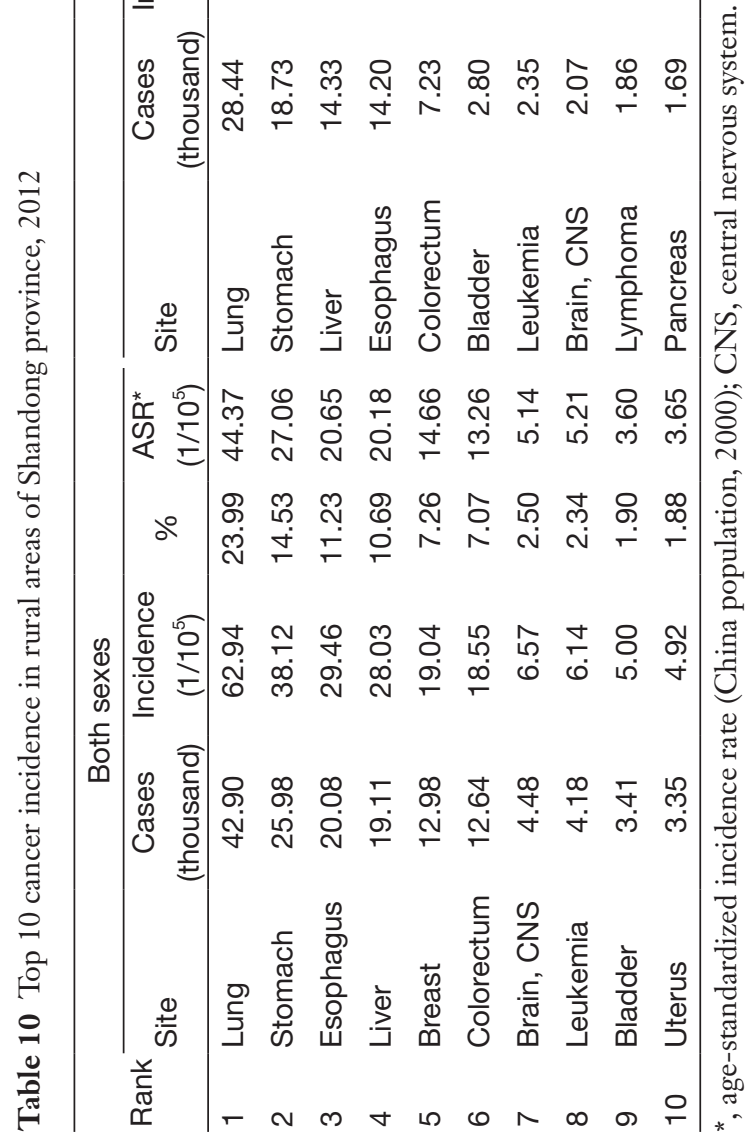

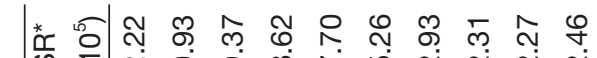
का

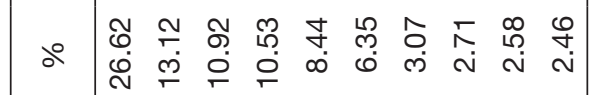

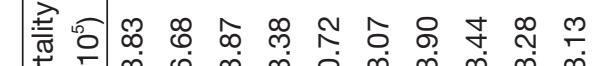

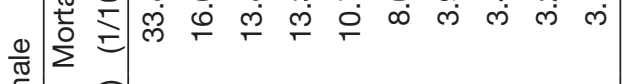
है

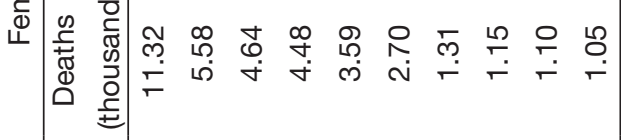

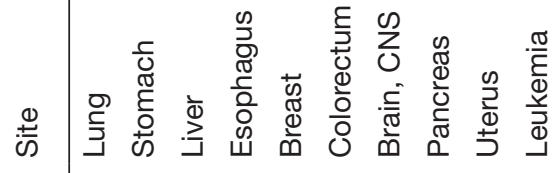
*

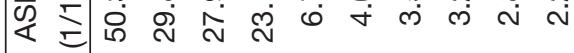

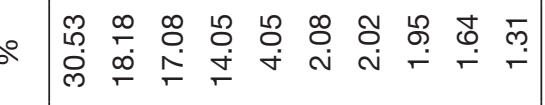

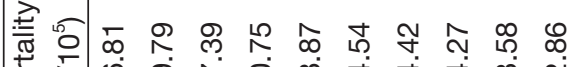
$\frac{\infty}{\sqrt{\pi}}$ 高 $\sum^{\pi}$

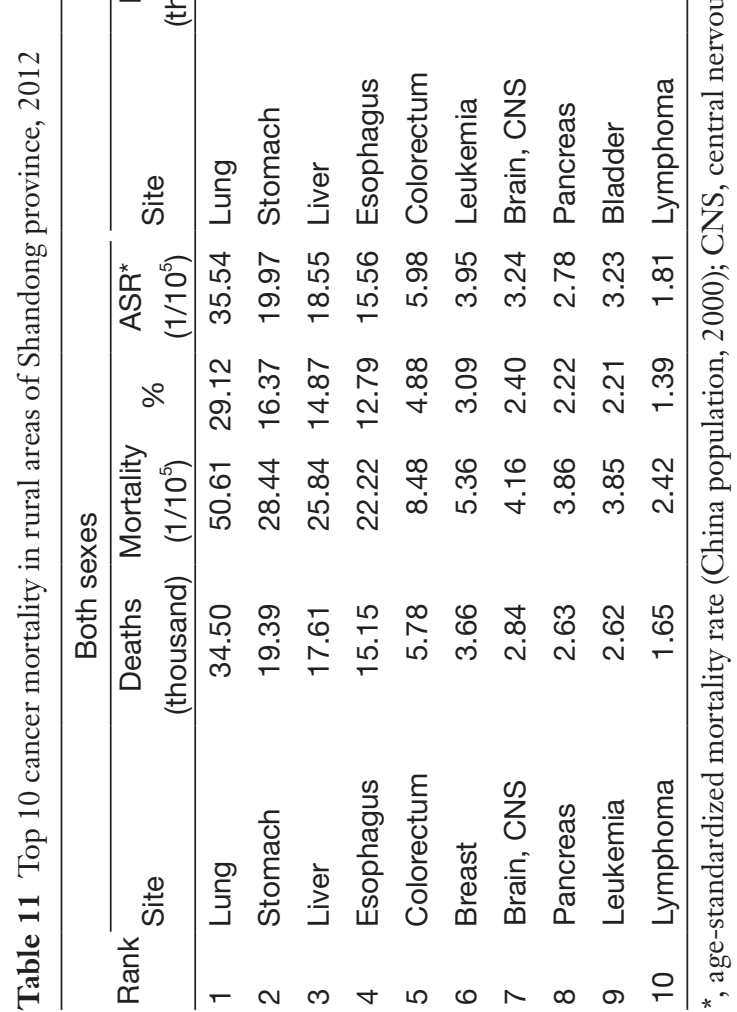


and the leading cause of death both in urban and rural areas of Shandong province, which was similar to that of China. Cigarette smoking, radon, secondhand smoke exposure and air pollution would be major factors associated with lung cancer $(13,14)$. Effective smoking control has been shown to reduce lung cancer incidence in the US since 1999 (15). Household air pollution may be the main reason for lung cancer in rural areas (16). A large population-based case-control study found that good ventilation in the kitchen and bedroom was inversely associated with lung cancer risk (17). Breast cancer and colorectal cancer are more common in urban, mainly because more people living in urban areas have a western lifestyle and physical inactively. In urban females, thyroid cancer and cervical cancer had ranked sixth and seventh, respectively, which needs to pay more attention. Cancers in upper digestive system areas are till the frequent cancers in rural areas, and health education, promotion and early detection should be placed priority in cancer strategies. Based on the current situation, different strategies should be implemented in different regions. Attention should be paid not only to the cancer with high incidence and mortality rates, but also to the cancer with rapid increasing trend. According to the population-based survival study in China, survival in rural areas was only half of that in urban areas (18), the main reason may be poor quality of cancer care and limited medical treatment for patients living in rural China. The government should balance the medical resources and take effective measures to bridge the gap.

With the development of economies and changes associated with urbanization, as well as the growth and population aging, the incidence and mortality keep increasing in China $(19,20)$. Targeted prevention, early detection and treatment programs should be carried out by health apartment to control the increasing cancer burden.

\section{Acknowledgements}

We gratefully acknowledged the cooperation of all the population-based cancer registries in Shandong province in providing cancer statistics, data collection, sorting, verification and database creation. At the same time, we also acknowledged the guidance of the National Central Cancer Registry in work training, data analysis and processing. The authors assume full responsibility for analyses and interpretation of these data.

\section{Footnote}

Conflicts of Interests: The authors have no conflicts of interest to declare.

\section{References}

1. Torre LA, Bray F, Siegel RL, et al. Global cancer statistics, 2012. CA Cancer J Clin 2015;65:87-108.

2. Torre LA, Siegel RL, Ward EM, et al. Global Cancer Incidence and Mortality Rates and Trends-An Update. Cancer Epidemiol Biomarkers Prev 2016;25:16-27.

3. National Bureau of Statistics of China. China Statistical Yearbook, 2010. Beijing: China Statistics Press, 2010.

4. Parkin DM. The evolution of the population-based cancer registry. Nat Rev Cancer 2006;6:603-12.

5. Curado MP, Edwards B, Shin HR, et al. Cancer Incidence in Five Continents. Vol. IX. Lyon: IARC, 2008:1-837.

6. Felay J, Burkhard C, Whelan S, et al. Check and Conversion Programs for Cancer Registries. IARC Technical Report No.42. Lyon: IARC, 2005.

7. Ferlay J. The IARCcrgTools Programs. Lyon: IARC, 2006. Available online: http://www.iacr.com.fr/index. php?option=com_content\&view=category\&layout=blog\&i $\mathrm{d}=68 \&$ Itemid $=445$

8. Ellis L,Woods LM, Estève J, et al. Cancer incidence, survival and mortality: explaining the concepts. Int J Cancer 2014;135:1774-82.

9. Chen W, Zheng R, Zhang S, et al. Annual report on status of cancer in China, 2010. Chin J Cancer Res 2014;26:48-58.

10. Chen W, Zheng R, Zeng H, et al. Annual report on status of cancer in China, 2011. Chin J Cancer Res 2015;27:2-12.

11. Jung KW, Park S, Won YJ, et al. Prediction of cancer incidence and mortality in Korea, 2012. Cancer Res Treat 2012;44:25-31.

12. Chen W, Zheng R, Zhang S, et al. Report of cancer incidence and mortality in China, 2012. Zhongguo Zhong Liu (in Chinese) 2016;25:1-8.

13. Ridge CA, McErlean AM, Ginsberg MS. Epidemiology of lung cancer. Semin Intervent Radiol 2013;30:93-8.

14. Guo Y, Zeng H, Zheng R, et al. The association between lung cancer incidence and ambient air pollution in China: A spatiotemporal analysis. Environ Res 2016; 144:60-5.

15. Kort EJ, Paneth N, Vande Woude GF. The decline in U.S. cancer mortality in people born since 1925. Cancer Res 2009;69:6500-5.

16. Seow WJ, Hu W, Vermeulen R, et al. Household air pollution and lung cancer in China: a review of studies in Xuanwei. Chin J Cancer 2014;33:471-5.

17. Jin $Z Y, W u M, H a n R Q$, et al. Household ventilation may reduce effects of indoor air pollutants for prevention of lung cancer: a case-control study in a Chinese population. 
PLoS One 2014;9:e102685.

18. Zeng H, Zheng R, Guo Y, et al. Cancer survival in China, 2003-2005: a population-based study. Int J Cancer 2015;136:1921-30.

19. Chen WQ, Zheng RS, Zeng HM, et al. Trend analysis and projection of cancer incidence in China between 1989 and 2008. Zhonghua Zhong Liu Za Zhi (in Chinese) 2012;34:517-24.

20. Zeng HM, Zheng RS, Zhang SW, et al. Trend analysis of cancer mortality in China between 1989 and 2008. Zhonghua Zhong Liu Za Zhi (in Chinese) 2012;34:525-31.

Cite this article as: $\mathrm{Fu} \mathrm{Z,} \mathrm{Lu} \mathrm{Z,} \mathrm{Li} \mathrm{Y,} \mathrm{Zhang} \mathrm{J,} \mathrm{Zhang} \mathrm{G,}$ Chen X, Chu J, Ren J, Liu H, Guo X. Cancer incidence and mortality in Shandong province, 2012. Chin J Cancer Res 2016;28(3):263-274. doi: 10.21147/j.issn.1000-9604.2016.03.01 